\title{
IRNSS Orbit and Clock Bias Estimation using Navic Ground Receiver Data: Extended Kalman Filter
}

\author{
Varsha H. S., Shreyanka B. Chougule, N. V. Vighnesam, Sudha K. L.
}

\begin{abstract}
The aim of this work is to precisely estimate the IRNSS satellite's orbit and clock errors using NavIC receiver data. Orbit determination is required to precisely calculate the user/receiver position on the Earth. In this study, Bengaluru, Surat, Kolkata, and Hyderabad's NavIC ground receivers' data is considered for orbit estimation. The pseudo-range measurements received by the ground receivers have multiple errors added due to ionospheric delay, tropospheric delay, multipath delays, satellite clock errors, and some unmodeled effects. But, the major factor accounting for errors is the satellite clock error. Hence, along with position and velocity of the satellite, even the clock correction is estimated using Extended Kalman Filter (EKF). EKF is a sequential estimation algorithm which estimates satellite position, velocity and clock error at each time instant. In this paper, results of all seven IRNSS satellite's orbit determination are discussed.
\end{abstract}

Keywords: Clock bias, Estimation algorithms, Extended Kalman Filter, IRNSS, NavIC receiver, Orbit Determination, Satellite Position Estimation

\section{INTRODUCTION}

Indian Regional Navigation Satellite System (IRNSS) is India's own navigation system used to provide accurate real-time positioning and timing services over India and the region extending to 1,500 kilometers $(930 \mathrm{mi})$ around India. It is a seven satellite constellation consisting of three are in geo-stationary orbit, namely, IRNSS 1C, IRNSS $1 \mathrm{~F}$ and IRNSS $1 \mathrm{G}$ and four are in geo-synchronous orbit, namely, IRNSS $1 \mathrm{~B}$, IRNSS 1D, IRNSS $1 \mathrm{E}$ and IRNSS $1 \mathrm{I}$. The seven satellite constellation has an operational name of NavIC Navigation with Indian Constellation. The three satellites in the Geostationary orbit (GEO) are positioned at $32.5^{\circ}$ east, $83^{\circ}$ east and $131.5^{\circ}$ east longitude respectively. The other four satellites in the Geosynchronous orbit (GSO) will appear to be moving in the form an ' 8 '. Two cross equator at $55^{\circ}$ east and two at $111.75^{\circ}$ east. Fig. 1 shows the ground track plot of

Revised Manuscript Received on December 30, 2019.

* Correspondence Author

Varsha H. S.*, Dayananda Sagar College of Engineering, Bengaluru 560078 (Karnataka) India. E-mail: varshahs29@gmail.com

Shreyanka B. Chougule, Dayananda Sagar College of Engineering, Bengaluru 560078 (Karnataka) India. E-mail: shreyankabc594@gmail.com

Dr. N. V. Vighnesam, Dayananda Sagar College of Engineering, Bengaluru 560078 (Karnataka) India. E-mail: vighnesam@gmail.com

Dr. Sudha K. L., Dayananda Sagar College of Engineering, Bengaluru 560078 (Karnataka) India. E-mail: klsudha1@rediffmail.com

(C) The Authors. Published by Blue Eyes Intelligence Engineering and Sciences Publication (BEIESP). This is an open access article under the CC BY-NC-ND license (http://creativecommons.org/licenses/by-nc-nd/4.0/) the seven IRNSS satellites.

One of the major applications of navigational systems is to determine the correct position of the user/ ground station positioned on the Earth [1]. In order to achieve accurate user position, it is first required to know the precise orbit of the navigational satellites.

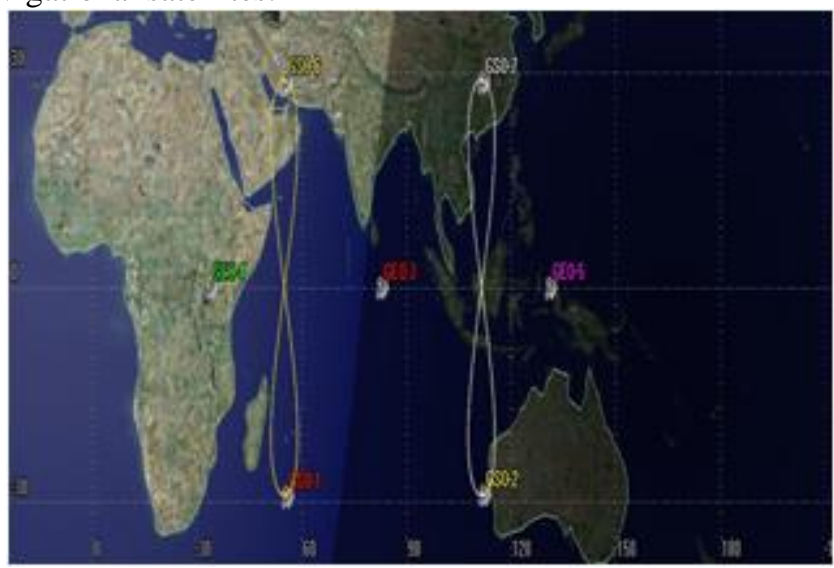

Fig.1. Ground track plot of IRNSS satellite's

Satellite orbit determination (OD) estimates the position and velocity of a satellite orbiting the Earth. The future state of motion of a satellite is estimated by considering the past observations. This is known as satellite orbit propagation. All the satellites in the navigational system have precise Rubidium or Cesium stable atomic clocks. IRNSS receivers have crystal oscillator which should be synchronized with the onboard atomic clock. But, the receiver uses economical kind of clock which is not exactly synchronized with the onboard satellite clock. Therefore, a clock offset is observed between the onboard satellite and the receiver clock [4].

The distance or range between the satellite and receiver is computed by measuring the time elapsed for a signal to propagate from a satellite to a receiver and multiplying it by the speed of light. This implies that any error in time will be reflected in the computed range. So the receiver clock error should be estimated. Availability, reliability and integrity of IRNSS navigation parameters are affected by satellite clock errors. Hence, satellite clock error needs to be corrected for precise navigation applications.

The ionosphere and troposphere are not uniform in composition and the refractive index changes all along the path of a signal. Change in signal speed changes the travel time of the signal and, therefore, changes the apparent pseudorange computed.

Published By:

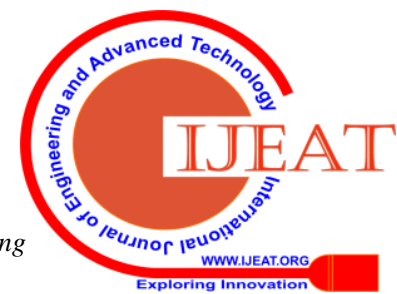


For precise navigation solution the pseudorange needs to be corrected for errors like satellite clock error, ionospheric delays, tropospheric delay, multipath delay etc. [3]. The errors, their effects on the pseudorange measurements and the necessary corrections to be made on the pseudorange measurements are explained in detail in [12].

Satellite Application Centre (SAC), ISRO installed Accord Software Pvt. Ltd. IGS (IRNSS/GPS/SBAS) receiver in the department of Electronics and Communication, Dayananda Sagar College of Engineering (DSCE), Bengaluru, India. Systematic and routine measurements are being carried out from the network of IRNSS satellites. The receiver is a multi-constellation, multi-frequency GNSS receiver which tracks 7 IRNSS satellites, 11 GPS satellites and 2 Satellite based augmented system (SBAS) satellites. Dual Frequency corrections provide real-time ionospheric corrections for further accuracy enhancements. The receiver has inbuilt models to estimate the tropospheric and ionospheric delays which are made available to the end-user. For the present study, along with Bengaluru data, NavIC data has been collected from multiple ground stations like Surat, Kolkata and Hyderabad.

In this work, an attempt to precisely determine the orbits of all the seven IRNSS satellites is made. Most commonly used estimation techniques are batch estimation and sequential estimation. In batch least squares estimation, an entire set of data/ measurement is processed altogether to improve an epoch state, whereas, in sequential estimation process, the data/measurement is processed at every instant of time and the state vector is estimated for every measurement.

In this paper, EKF is used for orbit estimation and the algorithm is explained in section II. The methodology adopted for orbit estimation of IRNSS satellites is described in section III, followed by results in section IV.

\section{EXTENDED KALMAN FILTER}

The EKF algorithm is a two-step algorithm. The state model is estimated using the initial conditions and current measurement by recursively applying the algorithm. By having the initial state, state noise covariance and measurement noise covariance, filtering can be started. The input state transition function and measurement function are to be linearized in the initial step to obtain the state transition matrix and observation matrix. When the current measurement is available, it is compared with the predicted measurement and the residual is computed. Kalman gain is used to estimate the current state and it's covariance. The algorithm is explained in detail in [13].

A short description of continuous time low dynamics noise model developed in this project is briefed below. The dynamic disturbance noise (Process noise) covariance matrix $\mathrm{Q}$ is given as

$$
\mathrm{Q}_{\mathrm{b}}=\left[\begin{array}{cc}
(S f * T)+\frac{T^{3}}{3} * S g & \frac{T^{2}}{2} * S g \\
\frac{T^{2}}{2} * S g & S g * T
\end{array}\right]
$$

where Sf and Sg are power spectral densities of continuous time, process-time. The Sf, Sg and the measurement noise covariance ' $R$ ' are set arbitrarily from the range of values provided from UR Rao Satellite Centre, ISRO, Bengaluru.
' $\mathrm{T}$ ' is the sampling period. The receiver range measurement error standard deviation is fixed to $\sigma=10$.

$$
\mathrm{Q}_{\mathrm{xyz}}=\sigma^{2} *\left[\begin{array}{cc}
\frac{T^{3}}{3} & \frac{T^{2}}{2} \\
\frac{T^{2}}{2} & T
\end{array}\right]
$$

The covariance matrix of process noise is given by

$$
\mathrm{Q}=\left[\begin{array}{cccc}
Q x y z & 0 & 0 & 0 \\
0 & Q x y z & 0 & 0 \\
0 & 0 & Q x y z & 0 \\
0 & 0 & 0 & Q b
\end{array}\right] \text { (3) }
$$

Time interval between two observations, $\mathrm{T}$, plays a vital role in calculating the Kalman gain. When there is a discontinuity in satellite signal's tracking from the receiver, the data gets lost for that particular instant. Data discontinuity can be caused by various reasons like power loss, very low signal-to-noise ratio, a failure of the receiver software, a malfunctioning satellite oscillator, severe ionospheric conditions and obstructions from surroundings. Under such circumstances, when the satellite's signal reappears, the tracking resumes. The software program has been developed in such a manner that, it reads the date and time of each signal and whenever there's a discontinuity, it automatically calculates time interval ' $\mathrm{T}$ ' and hence not disturbing the estimation process.

Some advantages of Kalman filter are it is accurate, fast etc. when compared to differential correction algorithm, it is suitable for real time application because of recursive nature. Usually the state estimated by EKF is stable because the variance doesn't increase infinitely.

\section{METHODOLOGY}

The NavIC Receiver provides the satellite ephemeris, Ground station positions, Pseudorange measurements between the satellites and ground stations, Ionospheric correction, Tropospheric correction, Satellite clock correction etc,. The satellite Ephemeris and ground station position will be in Earth Centered Earth Fixed (ECEF) frame format. These are converted from ECEF to Earth Centered Inertial (ECI) frame format.

The observed pseudorange is corrected using ionospheric and tropospheric error corrections provided by the NavIC receiver. Initial satellite state vector, four widely spread ground stations' position along with their respective corrected pseudoranges is input to the EKF algorithm.

The Extended Kalman Filter estimates the satellite's position, clock bias and clock drift at every time instant. The estimated satellite state vector $(\mathrm{x}, \mathrm{y}, \mathrm{z}, \dot{\mathrm{x}}, \dot{\mathrm{y}}, \dot{\mathrm{z}})$ is compared with the true satellite ephemeris to check the correctness of the program. Similarly, the estimated clock bias value is compared with the true satellite clock correction that is provided by the receiver. Hence, by comparing the estimated state with the true values, error in estimated state is found. The block diagram of the methodology is given in Fig. 2. 


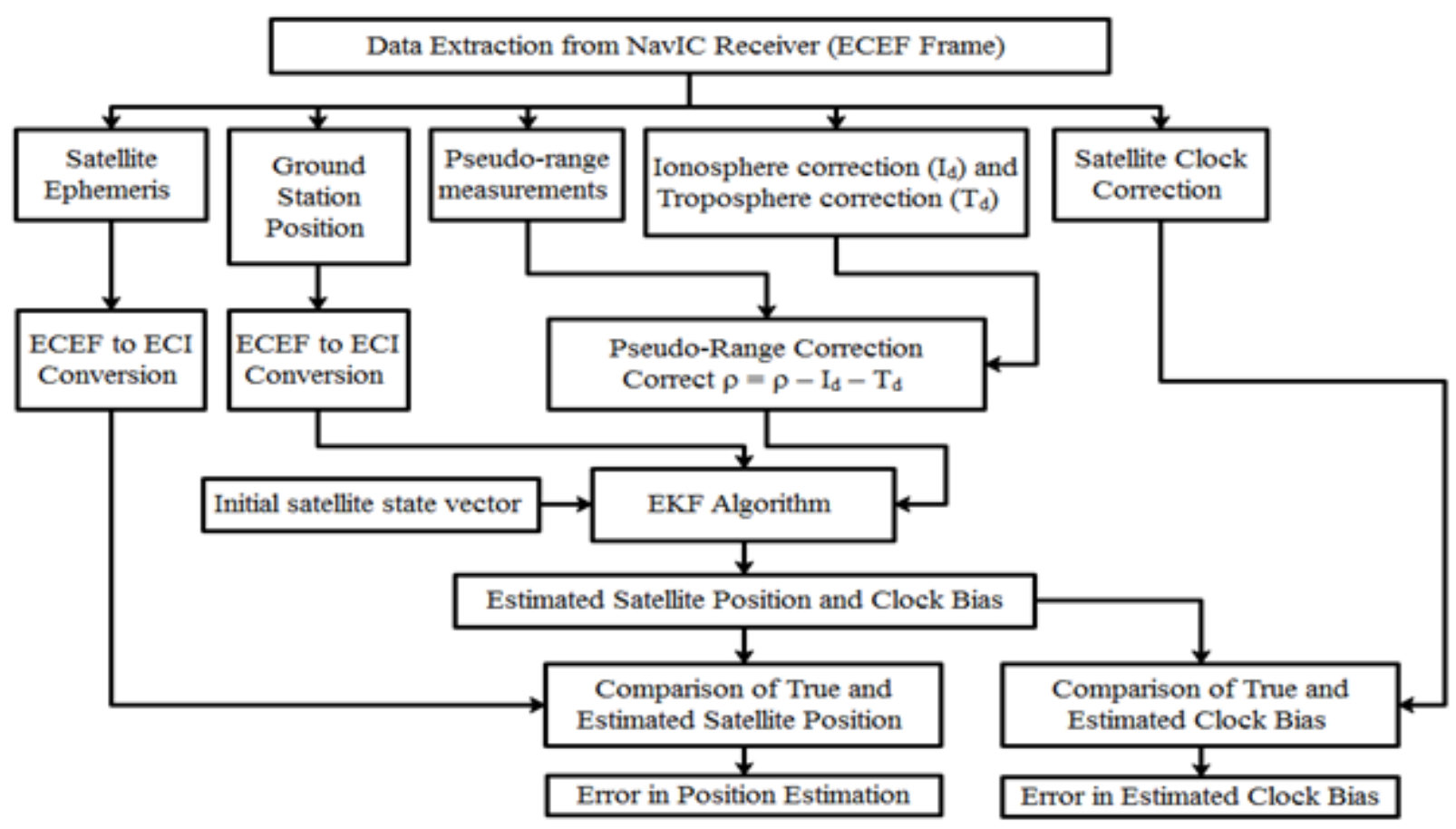

Fig. 2.Block diagram of methodology adopted

\section{OBSERVATIONS AND RESULTS}

Orbit determination (OD) is done for 7 IRNSS satellites, namely, IRNSS 1B, IRNSS 1C, IRNSS 1D, IRNSS 1E, IRNSS 1F, IRNSS $1 \mathrm{G}$ and IRNSS I1 and their respective results are discussed in this section. NavIC data is collected for the below mentioned time duration from four widely spread ground stations.

Start Date and Time : February 1, 2019 07:00:00 hours

End Date and Time $\quad$ : February 2, 2019 04:59:59 hours

Total Duration for OD: 22 hours

Time Interval $\quad: 1$ second

Ground stations : Surat, Bengaluru, Kolkata, Hyderabad

Table-I. Ground station positions

\begin{tabular}{|l|l|l|l|}
\hline $\begin{array}{l}\text { Ground } \\
\text { Stations }\end{array}$ & Latitude & Longitude & Altitude \\
\hline Surat & $\begin{array}{l}21.161981 \mathrm{de} \\
\text { g N }\end{array}$ & $\begin{array}{l}72.784043 \mathrm{de} \\
\text { g E }\end{array}$ & $\begin{array}{l}6368959491 . \\
4 \mathrm{~m}\end{array}$ \\
\hline Kolkata & $\begin{array}{l}23.239360 \mathrm{de} \\
\text { g N }\end{array}$ & $\begin{array}{l}87.852180 \mathrm{de} \\
\text { g E }\end{array}$ & $\begin{array}{l}6368437228 . \\
9 \mathrm{~m}\end{array}$ \\
\hline $\begin{array}{l}\text { Hyderaba } \\
\text { d }\end{array}$ & $\begin{array}{l}17.391225 \mathrm{de} \\
\text { g N }\end{array}$ & $\begin{array}{l}78.319155 \mathrm{de} \\
\text { g E }\end{array}$ & $\begin{array}{l}6370362900 . \\
5 \mathrm{~m}\end{array}$ \\
\hline Bengaluru & $\begin{array}{l}12.907795 \mathrm{de} \\
\text { g N }\end{array}$ & $\begin{array}{l}77.565736 \mathrm{de} \\
\text { g E }\end{array}$ & $\begin{array}{l}6371538573 \\
\mathrm{~m}\end{array}$ \\
\hline
\end{tabular}

Orbit estimation is done with actual initial state as well as with crude initial state (assuming precise initial state is unknown). The pseudorange values are corrected only for ionospheric and tropospheric delays. Hence, the pseudorange measurements contain satellite clock bias. The clock bias present in the measurements is estimated and compared with the true satellite clock error provided by NavIC receiver. Table II shows the satellite position and velocity error followed by the error in estimated clock bias for all seven satellites.

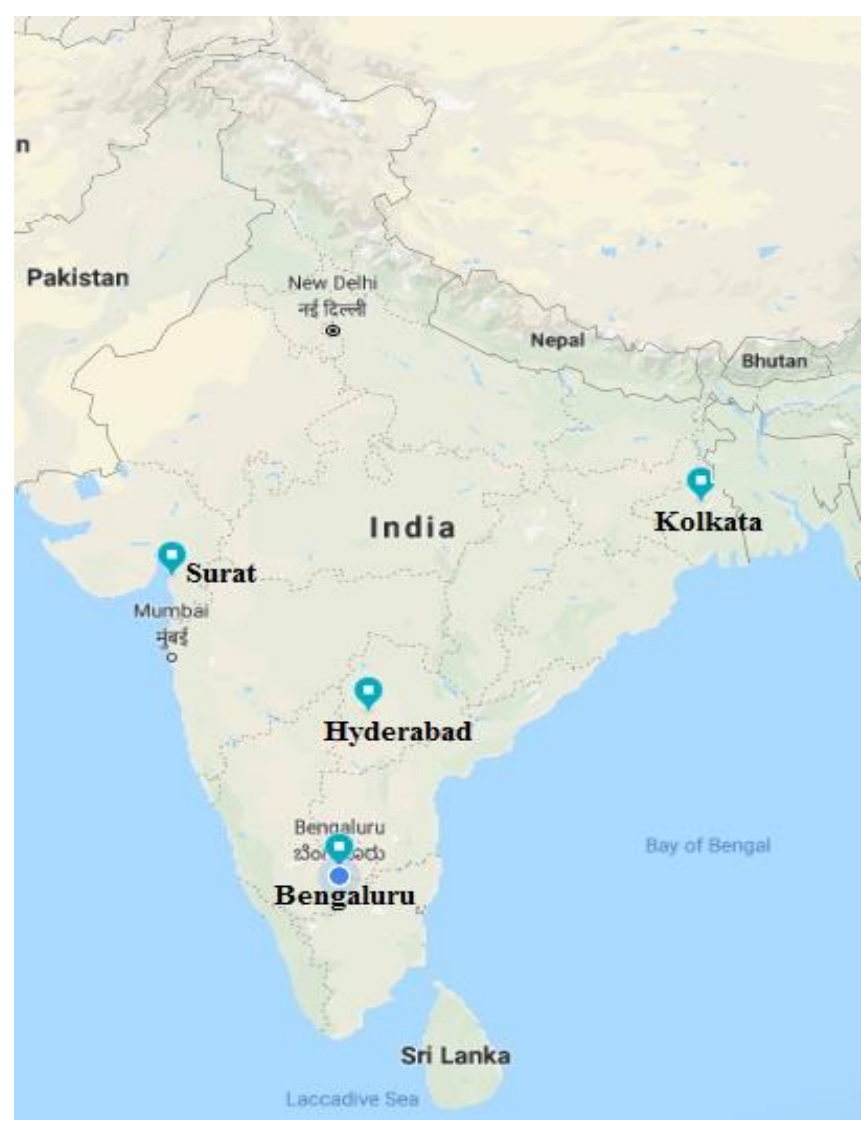

Fig. 3.Ground stations

Published By:

Blue Eyes Intelligence Engineering

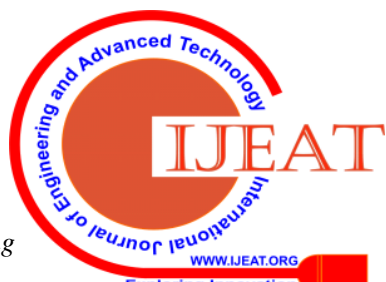


Table-II.Orbit Estimation of IRNSS satellites

\begin{tabular}{|c|c|c|c|c|c|c|c|c|c|c|}
\hline \multirow{2}{*}{ SATELLITE } & \multirow{2}{*}{$\begin{array}{l}\text { ORBIT } \\
\text { TYPE }\end{array}$} & \multicolumn{3}{|c|}{ POSITION ERROR (m) } & \multicolumn{3}{|c|}{ VELOCITY ERROR $(\mathrm{m} / \mathrm{s})$} & \multicolumn{3}{|c|}{ CLOCK BIAS (m) } \\
\hline & & AVERAGE & \begin{tabular}{|l|} 
STANDARD \\
DEVIATION
\end{tabular} & VARIANCE & AVERAGE & \begin{tabular}{|l|} 
STANDARD \\
DEVIATION
\end{tabular} & VARIANCE & ACTUAL & ESTIMATED & $\begin{array}{l}\text { AVERAGE } \\
\text { ERROR }\end{array}$ \\
\hline IRNSS 18 & GSO & 4.35549006 & 5.30355867 & 28.127735 & 0.2564601 & 0.81908966 & 0.67090787 & 111446 & 111441.779 & -4.2205016 \\
\hline IRNSS $1 \mathrm{C}$ & GEO & 2.33467336 & 0.7608234 & 0.5788523 & 0.1901083 & 0.16196749 & 0.02623347 & 142897 & 142894.929 & -2.07133186 \\
\hline IRNSS 10 & GSO & 3.33631896 & 0.62232233 & 0.3872851 & 0.293872 & 0.05904595 & 0.00348642 & 80363 & 80359.9118 & -3.08819245 \\
\hline IRNSS IE & GSO & 2.93367177 & 0.6702096 & 0.4491809 & 0.2269392 & 0.03385177 & 0.00114594 & 165881 & 165878.314 & -2.68605082 \\
\hline IRNSS $1 \mathrm{~F}$ & GEO & 4.31960722 & 0.76254539 & 0.5814755 & 0.2769308 & 0.07459989 & 0.00556514 & 87727 & 87722.9828 & -4.01720427 \\
\hline IRNSS $1 \mathrm{G}$ & GEO & 4.4721514 & 2.21760391 & 4.9177671 & 0.2772416 & 0.07019357 & 0.00492714 & 87727 & 87722.7856 & -4.21440371 \\
\hline IRNSS 11 & GSO & 3.24456123 & 4.21622264 & 17.776533 & 0.2371022 & 0.69319543 & 0.48051991 & 162735 & 162731.879 & -3.12095492 \\
\hline
\end{tabular}

\section{CONCLUSION}

IRNSS is India's own navigational system. One of the major applications of navigational systems is to determine the correct position of the user/ ground station positioned on the Earth. In order to achieve accurate user position, it is first required to know the precise orbit of the navigational satellites. Orbit estimation of all the seven satellites is done using Extended Kalman Filter algorithm. The noise variance matrices adopted for this project and the values of noise power spectral density and standard deviation of pseudorange measurements considered are discussed in section II.

The software program has been developed in such a manner that whenever there's a discontinuity in procuring data from satellite, it automatically calculates time interval ' $\mathrm{T}$ ' between the present data and the previous data at each instant and updates the same, which is then used in calculating the Kalman gain. In this manner, the estimation process is not disturbed. The error in estimated satellite position, velocity and clock bias is obtained by comparing the estimated values with their respective true values. Table-II given in section IV gives a summary of all the results obtained.

The orbit error obtained now is around $2 \mathrm{~m}-5 \mathrm{~m}$. Further, an attempt can be made to modify the algorithm or to adopt a new algorithm to improve the orbit estimation accuracy.

\section{ACKNOWLEDGMENT}

The work undertaken in this paper is supported by SAC/ISRO under NavIC GAGAN utilization program (Project ID: NGP 27), at Space Application Centre, Ahmedabad. Authors wish to acknowledge the support given by SAC/ISRO.

\section{REFERENCES}

1. Montenbruck O. and Gill E, "Satellite Orbits, Models, Methods and Applications”, Springer, 2001.

2. Montenbruck, Oliver \&Steigenberger, Peter \& Riley, Stuart. (2015). IRNSS Orbit Determination and Broadcast Ephemeris Assessment.

3. M. Pratap and E. Per, "Global Positioning System: Signals, Measurements and Performance," 2nd Edition, Ganga-Jamuna Press, New York, 2006.

4. BharatiBidikar, GottapuSasibhushanaRao, Laveti Ganesh, MNVS Santosh Kumar, "Satellite Clock Error and Orbital Solution Error Estimation for Precise Navigation Applications", (http://www.scirp.org/journal/pos), Positioning, 2014, 5, 22-26..
5. KaithaRajaiah, Manamohan Kamath, PrasantaMula, S. Nirmala, A. S. Ganeshan, S. C. Rathnakara, "Precise Orbit Determination of IRNSS 1A satellite using Laser ranging measurements", ISRO Satellite Center Journal of Spacecraft Technology, vol. 26, no. 2, 2015.

6. A. S. Ganeshan, S. C. Rathnakara, R. Gupta, A. K. Jain, "Indian Regional Navigation Satellite System (IRNSS) Concept", ISRO Satellite Center Journal of Spacecraft Technology, vol. 15, no. 2, 2005.

7. ISRO (2017) "IRNSS Signal In Space ICD forStandard Positioning Service”, v. 1.1, June 2017, ISROIRNSS-ICD-SPS-1.1, Indian Space Research Organization, Bangalore

8. Y. He, R. Martin and A. M. Bilgic, "Approximate iterative Least Squaresalgorithms for GPS positioning," The 10th IEEE International Symposium on Signal Processing and Information Technology, Luxor, 2010, pp. 231-236.

9. Sarunic,Peter W, Defence Science and Technology Group Edinburgh Australia, "Development of GPS Receiver Kalman Filter Algorithms for Stationary, Low-Dynamics, and High-Dynamics Applications", 2016. Online: https://apps.dtic.mil/dtic/tr/fulltext/u2/1010622.pdf

10. Accord Software and System Pvt. Ltd., "IRNSS/GPS/SBAS Receiver: User Manual", issue: 1.2, 11/02/2016.

11. Babu R, PrasantaMula, S. C. Ratnakara\& A S Ganeshan," IRNSS Satellite Parameter Estimation using Combination Strategy", Global Journal of Science Frontier Research: A Physics and Space Science, Volume 15, Issue 3, Version 1.0, Year 2015.

12. Varsha H.S, Shreyanka B Chougule, Dr.N.VVighnesam, Dr. K.L Sudha, "Analysis of Pseudo-range Measurements Observed in NavIC Receiver" International Journal of Engineering Research and Applications (IJERA), vol. 8, no.12, 2018, pp 43-46.

13. Varsha, H.S., Shreyanka, B.C., Vighnesam, N.V., Sudha, K.L.: IRNSS Position Estimation using Iterative Least Squares and Extended Kalman Filter, International Conference on Advanced Technologies in Intelligent Control, Environment, Computing and Communication Engineering (ICATIECE), 2019.

\section{AUTHORS PROFILE}

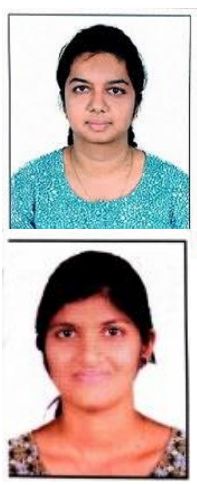

Varsha H. Sis currently working as a Junior Research Fellow for an ISRO Funded Project in Dayananda Sagar College of Engineering, Bengaluru. She graduated B.E in Electronics and Communication from Vidyavardhaka College of Engineering, Mysuru, in 2016 and M.Tech in Digital Electronics and Communication from Dayananda Sagar College of Engineering, Bengaluru, in 2018.

Shreyanka B. Chouguleis currently working as Junior Research Fellow for an ISRO Funded Project in Dayananda Sagar College of Engineering, Bengaluru. She graduated B.E in Electronics and Communication from KLS Gogte Institute of Technology, Belagavi, in 2016 and M.Tech in Digital Electronics and Communication from Dayananda Sagar College of Engineering, Bengaluru, in 2018. 


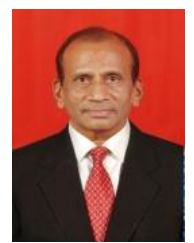

Dr. N. V. VighnesamDr.N.V.Vighnesam obtained his Ph.D degree in Mathematics from Indian Institute of Technology, Bombay and joined ISRO Satellite Centre, Bangalore in 1980. He was Head, Orbit Dynamics Division, Flight Dynamics Group. He worked on satellite Tracking data processing; satellite orbit prediction, determination, orbit simulations and related analysis. He was responsible for design and development of orbit determination system for all ISRO Satellites. He was actively involved in design and development of India's first Moon (Chandrayaan-1) mission and Mars mission (MOM) on orbit dynamics activities in addition to on-going missions. Currently he is working as a Professor in Department of Mathematics, Dayanandasagar College of Engineering since July 2014 and also the Principal Investigator (PI) for ISRO's funded project on NavIC (Navigation - Indian Constellation).

He was visiting scientist in the Federal Republic of Germany to work on satellite Preliminary Orbit Determination at GSOC during 1990-91. He has obtained ISRO Excellence Awards 2008 on 'Chandrayaan-1' and INSAT missions. He was International Programme Committee (IPC) Member for International Astronautical Federation (IAF), International ESA Conference on GNC, ESA's Small Satellites Systems and Services Symposium (4S Symposium). He has published more than 70 technical papers in national and international journals / symposiums.

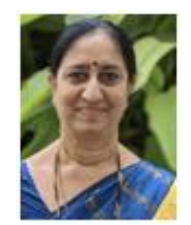

Dr. K. L.Sudha, presently working as professor in the Department of Electronics and Communication Engineering, Dayananda Sagar College of Engineering, Bangalore, India has 23 years of teaching experience in distinct Engineering Colleges. She obtained her Bachelor's degree in Electronics Engineering from Mysore University and Masters from Bangalore University. She obtained Ph.D for her work on "Detection of FH CDMA signals in time varying channel" from Osmania University, Hyderabad. Four scholars got awarded with Ph.D under her guidance and currently 6 scholars are working under her. She has guided many UG and PG students for their final year projects. She has published more than 85 research papers in national / International journals and conferences. She has also procured and executed few funded projects successfully. Her research interests include Wireless communication, coding theory, image processing and chaotic theory. 